\title{
Orexin A in Cortical Cultures: Expression and Effect on Synaptogenesis During Development
}

\author{
Irina I. Stoyanova - Wim L. C. Rutten • \\ Joost le Feber
}

Received: 2 May 2011/Accepted: 24 June 2011/Published online: 8 July 2011

(c) The Author(s) 2011. This article is published with open access at Springerlink.com

\begin{abstract}
Orexin A (OXA) is an excitatory hypothalamic neurotransmitter and ligand for Orexin Receptor-1 (OR1), isolated from a small group of hypothalamic neurons. OXA orchestrates different brain functions, and at the cognitive level some of the effects of insufficiency of OXA are wellknown, for example in Parkinson's disease. It is widely assumed that deteriorated cognitive processes are related to impaired network connectivity. However, little is known about the effects of OXA in network connectivity and synaptogenesis. Therefore, to obtain insight into this problem we designed experiments with two groups of networks of dissociated cortical neurons: one group incubated in a plain medium and another chronically treated with OXA. After 1, 2, 3 or 4 weeks in vitro we applied immunocytochemistry for detection of OXA, OR1, and synaptic marker synaptophysin. Shortly after plating, $91 \pm 8 \%$ of the neurons cultivated in a plain medium expressed OXA-immunoreactivity, which does normally not occur in vivo indicating that neurons may change their phenotype under non-natural culture conditions to develop synaptically coupled networks. The fraction of orexinergic neurons decreased to $33 \pm 21 \%$ after 4 weeks in vitro. OXA expression was highest in the first week of network formation, the period of maximum synaptogenesis, and then decreased and stabilized in the weeks thereafter. Our
\end{abstract}

I. I. Stoyanova $(\square)$ - W. L. C. Rutten · J. le Feber

Neural Engineering Department, Institute for Biomedical

Engineering and Technical Medicine MIRA, University of

Twente, BSS, ZH 226, P. O. Box 217, 7500, AE, Enschede,

The Netherlands

e-mail: stoyanovai@yahoo.co.uk

I. I. Stoyanova

Science Department, Roosevelt Academy, University of Utrecht, 4330, AB, Middelburg, The Netherlands hypothesis that OXA plays a role in the network development as a synaptogenic factor was supported by higher levels, earlier onset, and sustained increase of synaptophysin expression in experiments with chronic OXA application to the culture medium.

Keywords Orexin A - Synaptophysin - Synaptogenesis · Dissociated cortical neuronal cultures .

Immunocytochemistry · Rat

\section{Introduction}

Orexin A (OXA) is produced by a small group of neurons specifically located within and around the lateral hypothalamus (LH) (de Lecea et al. 1998). Like OXB, OXA is a neuropeptide encoded by mRNA which accumulates primarily after the third postnatal week (Gautvik et al. 1996). OXA is a 33 -amino acid peptide, the sequence of which is identical in human, rat, mouse, and bovine (Sakurai et al. 1998). Experiments with synaptically coupled rat hypothalamic neurons, demonstrate that Orexin acts as an excitatory neurotransmitter with two specific receptors (de Lecea et al. 1998), Orexin Receptor-1 (OR1) and Orexin Receptor-2 (OR2), which belong to the G-protein coupled receptor superfamily (Sakurai et al. 1998). These receptors share 64\% identity in their amino acid sequence and exhibit marked differential distribution. OXA is a selective ligand for OR1. In contrast to orexinergic neurons, the presence of ORs is not restricted to the hypothalamus. Both OR types have a high level of expression in multiple brain regions (Trivedi et al. 1998) and thus orexins may orchestrate a large number of processes in the nervous system (Fadel et al. 2002).

It is widely assumed that deteriorated cognitive processes are related to impaired network connectivity and 
activity. Conversely, several neurodegenerative diseases that lead to deteriorated cognitive abilities have been related to OXA deficiency. Both the number of orexinergic neurons in the hypothalamus and the levels of orexin in the cerebrospinal fluid are reduced by $72 \%$ in the end-stage in a mouse model of Huntington's disease (Petersén et al. 2005). Recent studies in Parkinson's patients found a $40 \%$ lower OXA concentration in the frontal cortex and a $25 \%$ reduction in the cerebrospinal fluid (Fronczek et al. 2007). These findings suggest that orexin may influence network connectivity and activity.

Experiments in vivo (Peever et al. 2003) and in vitro with acute application of the transmitter on organotypic brain slices (Horvath et al. 1999) or dissociated neuronal cultures (Stoyanova et al. 2011) indicated that OXA has an excitatory effect on network activity. However, the effects of OXA on cortical development remain unclear, in particular the effect on synaptogenesis.

It has been shown that synaptic formation responds to various inputs. For instance, Horvath and Gao (2005) demonstrated synaptic re-organization in orexinergic neurons in vivo after glucose deficiency, but detailed data about the synaptogenesis is not easy to acquire in vivo. Furthermore, it is very difficult to quantify orexinergic input to the cortex in vivo. Therefore, we used cultures of dissociated cortical neurons to investigate the effects of OXA on synaptogenesis in a controlled study.

Dissociated cortical neurons have been shown to form networks and to become spontaneously active after approximately a week (Van Pelt et al. 2004; Chiappalone et al. 2006). We designed experiments with two groups of networks: one incubated in plain medium, and the other in medium chronically supplemented with OXA. Immunostaining for OR1 was applied in order to determine at what developmental stage the receptor is expressed and neurons could react to the OXA application. To quantify the alterations in network formation we combined it with immunocytochemistry for detection of OXA and synaptic marker synaptophysin during the first 4 weeks of development in vitro.

\section{Materials and Methods}

\section{Dissociated Cell Cultures}

Newborn Wistar rats were anesthetized with ether and decapitated. The brains were removed and placed in RPMImedium. The meninges were removed; the cortices were dissociated and collected in chemically defined R 12 culture medium (Romijn et al. 1984) with trypsin for chemical dissociation. After the trypsin, $150 \mu \mathrm{l}$ of soybean trypsin inhibitor and $125 \mu \mathrm{l}$ of DNAse I (20.000 units, Life
Technology) were added, followed by mechanical dissociation of the neurons. The suspension was centrifuged at $1200 \mathrm{rpm}$ for $5 \mathrm{~min}$; the pellet was plated on glass cover slips pre-coated with $20 \mathrm{mg} / \mathrm{ml}$ poly-ethylene-imine (Fluka, Buchs, Switzerland), at a density of approximately 3000 cells $/ \mathrm{mm}^{2}$. They were allowed to attach for $2 \mathrm{~h}$ at $37^{\circ} \mathrm{C}$ and $5 \% \mathrm{CO}_{2}$ in air and kept in $\mathrm{R} 12$ medium optimized with $50 \mathrm{ng} / \mathrm{ml}$ nerve growth factor (Invitrogen, Carlsbad, CA). Medium was serum-free to suppress glial cell proliferation and keep their concentration lower than 5\% (Romijn et al. 1984). It was renewed every 2-3 days. For assessment of the effect of OXA on synaptogenesis $2 \mu \mathrm{M}$ OXA was added to the medium. We use this concentration because it has been shown that it produces measurable changes in neuronal activity (Horvath et al. 1999). Cultures were fixed in $4 \%$ paraformaldehyde in $0.1 \mathrm{M}$ phosphate buffered saline (PBS), $\mathrm{pH} 7.4$ after 1, 4, 7, 14 or 21 DIV, and processed for immuno-detection of OXA, OR1, or synaptophysin. For identification of the cellular type we applied specific markers, i.e., anti-Microtubule Associated Protein (MAP2) antibody as a neuronal marker and anti-Glial Fibrillary Acidic Protein (GFAP) antibody, a marker for glial cells.

\section{Animals and Tissue Preparation}

Adult Wistar rats (250-359 g body weight) were used as a positive control for testing specificity of anti-OXA antibody. All housing facilities and procedures used were approved by the Animal Care and Use Committee of the University of Twente, and were carried out in accordance with the European Communities Council Directive of 24 November 1986. The rats were anesthetized and perfused with $4 \%$ paraformaldehyde in $0.1 \mathrm{M}$ PBS ( $\mathrm{pH}$ 7.4). The brains were removed, postfixed by immersion in the same fixative for $24 \mathrm{~h}$ at $4^{\circ} \mathrm{C}$ and cryoprotected in $25 \%$ sucrose in PBS at $4^{\circ} \mathrm{C}$. They were embedded in TissueTek OCT compound (Miles Inc., Elkhart, NI, USA), frozen and $30 \mu \mathrm{m}$ thick sections were cut in a cryostat at $-20^{\circ} \mathrm{C}$. After rinsing in $0.1 \mathrm{M} \mathrm{PBS}$, free-floating slices selected from -0.92 to $-5.20 \mathrm{~mm}$ (from Bregma), i.e., where the orexinergic neurons are localized, were processed immunocytochemically.

Immunocytochemistry

The immunostaining was performed according to the $\mathrm{ABC}$ (avidin-biotin-horseradish peroxidase) method (Hsu et al. 1981). Briefly, the endogenous peroxidase was inactivated with hydrogen peroxide $(0.3 \%$ in methanol/PBS for $30 \mathrm{~min}$ ), and the background was blocked with 5\% normal goat serum (NGS) and $1 \%$ bovine serum albumin (BSA) in $0.5 \%$ Triton X-100. Appropriate rinsing in PBS followed these and subsequent procedures. Incubation in the primary 


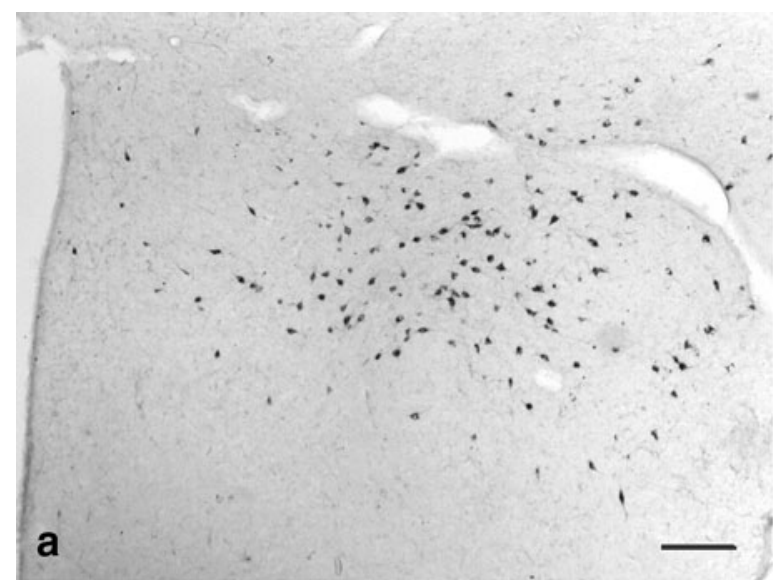

Fig. 1 Coronal section through the hypothalamus of an adult rat labeled with OXA antiserum. a Low magnification showing OXApositive neurons distributed bilaterally in the lateral hypothalamus

antibodies (rabbit anti-Orexin A IgG, Calbiochem, Merck Chemicals, Nottingham, dilution 1:500; goat anti-OR1, 1:200, AbD Serotec, Oxford, UK; mouse anti-synaptophysin, 1:800, Abcam, Cambridge, UK; rabbit anti-MAP2, 1:200, and rabbit anti-GFAP, 1:750, both Abcam) was for $20 \mathrm{~h}$ at room temperature (RT), followed by treatment for $2 \mathrm{~h}$ with biotinilated secondary antibodies (goat anti-rabbit, donkey anti-mouse, donkey anti-goat IgG (1:500); Jackson ImmunoResearch, West Grove, PA, USA). After rinsing, the cell cultures and brain slices were incubated for $1 \mathrm{~h}$ in ABC complex (Vector Labs, Burlingame, CA, USA, $6.25 \mu \mathrm{l} / \mathrm{ml}$ of each compound in PBS). The peroxidase activity was visualized using $2.4 \%$ SG substrate kit (Vector) in PBS for $5 \mathrm{~min}$ at room temperature. The specimens were counterstained with $0.5 \%$ Neutral red (Sigma, St. Louis, MO, USA). Finally, the cultures and the brain slices were dehydrated in alcohol, cleared in xylene and coverslipped with Entellan (Merck, Darmstadt, Germany).

Negative controls included incubation at $4^{\circ} \mathrm{C}$ for $24 \mathrm{~h}$ after antigen-antibody preabsorption with the native antigen OXA (Calbiochem, Merck Chemicals, Nottingham), and replacement of the other primary antibodies with normal serum.

\section{Data Analysis and Photomicrograph Production}

Seven culturing experiments were made; eight to ten brains were used for each experiment. The dissociated cells were plated on 120 coverslips per experiment. We semi quantitatively graded the level of OXA expression in the neurons according to Ljungdahl et al. (1978), using the following four-point intensity scale: (-) background intensity of staining; (+) low intensity; $(++)$ moderate intensity; $(+++)$ high intensity. The images were generated through $10 \times, 20 \times, 40 \times$, and $60 \times$ objectives and the cultures and

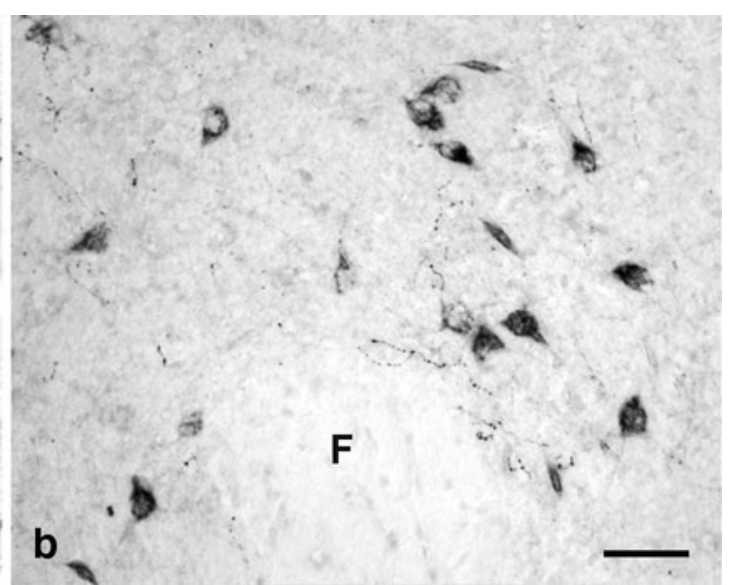

(dark cells). b Higher magnification of OXA-positive neurons in the perifornical area. Fornix (F). Scale bars: (a) $200 \mu \mathrm{m}$; (b) $50 \mu \mathrm{m}$

brain slices were photographed with a Nikon DS-Fil digital camera linked to a Nikon Eclipse 50i microscope. All digital images were matched for brightness in Adobe Photoshop 7.0 software. Cell counts for OXA expression were performed on five randomly chosen coverslips per age group. Statistical computations were performed using the StatView package for Windows, v 4.53 (Abacus Concepts Inc., Berkeley, California, USA). The analysis showed that data distribution is not normal, therefore we used the non-parametrical MannWhitney and Kruskal-Wallis tests for statistical comparison. For quantitative analysis of synaptic marker expression we counted the number of granules of reaction product after the synaptophysin staining. In each culture we calculated the granule density under a high magnification at seven different randomly chosen locations. We used Nikon NIS-Elements software, and obtained estimates of the mean densities per $\mu \mathrm{m}^{2}$ and standard deviations. We used two-way ANOVA to assess the statistical significance of density differences. Known sources of variation were control vs. OXA treated cultures and culture age. All data were presented as the mean $\pm \mathrm{SD}$ (standard deviation). A $P$ value smaller than 0.05 was considered statistically significant.

\section{Results}

\section{Specificity}

The immunoreactivity was readily discernible by the presence of a dark-gray immunoreactive product. Neuronal structures were considered to be immunopositive when their staining was stronger than that in the background. In the brain slices OXA-positive cell bodies were found bilaterally, confined to the lateral hypothalamus at the level of the median eminence (Fig. 1a, b). There were no neurons in the 
brain cortex expressing OXA. We saw no immunoreactivity for OXA, OR1 or synaptophysin in the controls after preabsorption of the antibody with the antigen or its replacement with normal serum at the same concentration.

Identification of Neuronal and Glial Cells in the Cultures

In order to exclude the opportunity for OXA to be expressed by glial cells we applied immunostaining for neuronal and glial markers MAP2 and GFAP, respectively. In 1-day-old cultures it was hard to morphologically discriminate neurons from glial cells, but immunolabeling for GFAP indicated a very low fraction of glial cells. After one or more weeks of culturing, glial cells were well developed and none of the OXA-labeled cells had their morphology. In contrast, immunostaining for MAP2 and OXA clearly showed the same morphology of the labeled cells, i.e., only neurons were OXA-positive (data not shown).

Expression of OXA-, OR1- and SynaptophysinImmunoreactivity

Immunolabeling revealed that $91 \pm 8 \%$ of the neurons in 1-day-old cultures were OXA-immunoreactive (IR). The reaction product was not uniformly distributed throughout the cytoplasm, but was sometimes concentrated in a restricted area of the perikaryon (Fig. 2a). The size of the neuronal cell bodies was small and the arborizations were shorter and thinner.

In cultures grown for 1 week (Fig. 2b), a significantly lower fraction of all neurons $(83 \pm 13 \%, P=0.03)$ were OXA-IR. The majority of orexinergic neurons were small, with fine bipolar or tufted dendrites, but some medium- to large-sized cells with basket-like morphology were also observed. The immunoreactivity was evenly distributed throughout the cell soma, excluding the nucleus.

In two-week-old cultures the proportion of OXA-IR neurons was $49 \pm 18 \%$, significantly lower than in 1-dayand 1 -week-old cultures $(P<0.0001)$. The neurons were already well developed and the orexinergic population consisted of two well distinguishable types of cells: spindleshaped neurons and multipolar, pyramidal neurons. The intensity of immunostaining varied from $(-)$, mainly in the large-sized perikarya, to $(+),(++)$, and $(+++)$ in the medium- to small-sized neurons of both multipolar and bipolar types (Fig. 2c). After 3 weeks' of growth (Fig. 2d), the clusters of neurons became very dense and the percentage of OXA-expressing neurons was $32 \pm 19 \%$. In 4-week-old cultures (Fig. 2e) the fraction of orexinergic neurons was not changed significantly: $33 \pm 21 \%$ as summarized in Fig. 2f.

Figure 3 shows the time course of expression of OR1-IR in cultures between 1 day and 4 weeks in vitro. After 1 day in vitro, neurons already demonstrated some initial expression of OR1 peptide (Fig. 3a). After 1 week, the immunoreactivity was more prominent and expressed in almost all neurons (Fig. 3b). When neurons were cultured for two or more weeks, the immunoreaction product was clearly discernible not only on the perikarya but also along the neurites (Fig. 3c, d).

Chronic treatment of the cultures with medium supplemented with $2 \mu \mathrm{M}$ OXA resulted in significant changes in synaptic development. As early as 4 days after OXA application, the neurons already showed some expression of synaptophysin (Fig. 4a), while the control cultures were immunonegative (Fig. 4b). After 2 weeks of incubation, the synaptophysin staining was much more abundant in conditioned cultures than in controls (Fig. 4c, d). Not only were the cell bodies immunolabeled, but the reaction product was also found along the neuronal arborizations. Three-week-old cultures were already well developed and equipped with synaptic contacts but comparison with controls showed substantial differences in synaptophysin expression (Fig. 4e, f). Figure 5 depicts the development of the synaptic density in control cultures and OXA treated cultures. Two-way ANOVA showed that the synaptophysin granule density increased significantly with age in all cultures $(P=0.0005)$ and that the OXA conditioned networks had a significantly higher density of synaptophysin granules than the controls $\left(P=1.5 \times 10^{-6}\right)$.

\section{Discussion}

This study demonstrates for the first time OXA expression in cultured dissociated cortical neurons. As is well-known, OXA is produced only by hypothalamic neurons and cortical neurons normally do not synthesize it in vivo neither in adult nor in newborn rats (Stoyanova et al. 2010). However, in this study, we found that isolated cortical neurons did produce OXA, which suggests a conditioningrelated change of phenotype. The initially high level of OXA expression decreased in the next 3-4 weeks of culturing, in close synchrony to the onset and subsequent increase of bioelectric activity.

Although neurotransmitters are generally associated with neuronal communication in the mature brain, many of them have been shown to influence several aspects of brain development and maturation (Levitt et al. 1997). The synthesis de novo of OXA in isolated neuronal cultures, which we demonstrated here, may suggest some role for OXA as a signaling molecule for network development as it has been previously revealed for GABA and glutamate (Spoerri 1988; Ben-Ari et al. 1994). The orexin system develops very early in vivo (Van den Pol et al. 2001; Stoyanova et al. 2010); therefore the early expression of 

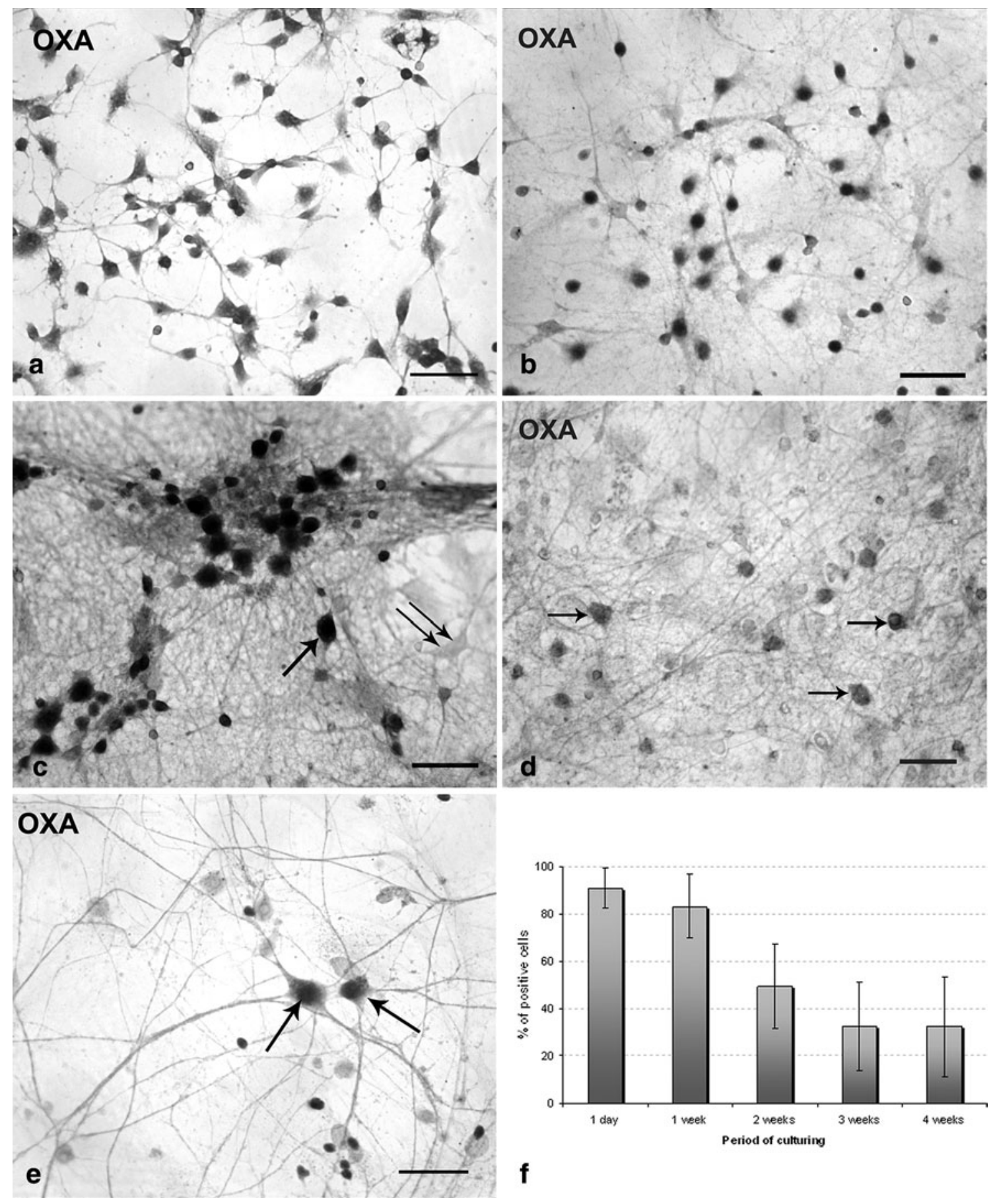

Fig. 2 a 1-Day-old culture processed for OXA detection. The neurons are underdeveloped, and most of them exhibit OXA-IR as dark granules, which are not dispersed in the entire cytoplasm. b OXA expression in 1-week-old culture. IR varies from negative, mainly in the large-sized perikarya, to highly intensive in the medium- to small-sized neurons. c Two-week-old culture. Cells and outgrowths are well developed. The thick arrow indicates a spindle-

OR1 which we observed already at 1 DIV could be another indication for the importance of orexinergic input in developing networks. This observation is in accordance with the findings that the human sleep disorder, narcolepsy,

shaped neuron exhibiting high intensity of OXA-staining. The double arrow is pointing to a pyramidal perikaryon, which is OXA-negative. d Moderate intensity of OXA-labeling of neuronal somata (arrows) in a 3-week-old culture. e Four-week-old culture. Two OXA-positive multipolar neurons (arrows) with thick non-varicose processes forming a solid network. f Histogram of the OXA expression after subsequent DIV. Scale bars: (a, c, e) $30 \mu \mathrm{m}$; (b, d) $50 \mu \mathrm{m}$

as well as different neurodegenerative diseases, were recently related to a massive reduction or deficit of orexins and their receptors and a decreased level of OXA in the cerebrospinal fluid (Cui et al. 2010; Friedman et al. 2007; 


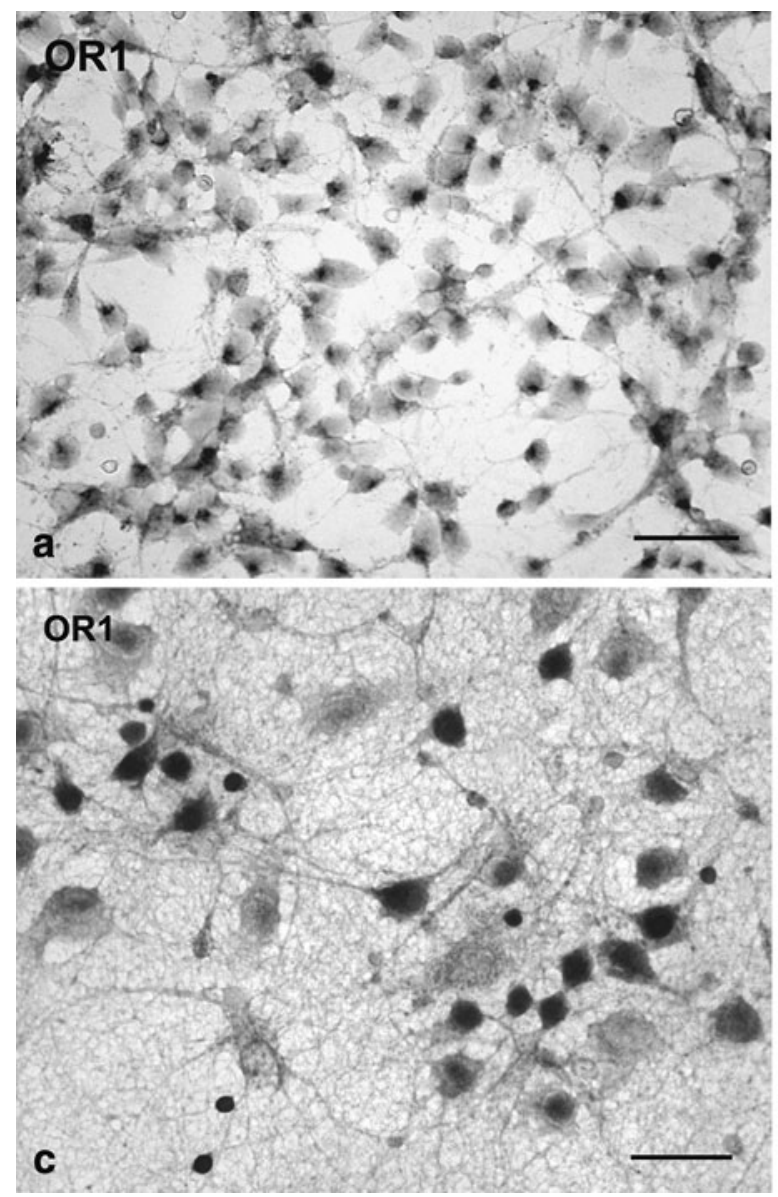

Fig. 3 a Neurons cultured for 1 day expressing some IR for OR1, mainly on the cell bodies. b Well developed neuronal network after 1 (b), 2 (c), and 4 (d) weeks in vitro. Most of the neurons express OR1,

Petersén et al. 2005; Ripley et al. 2001). Experiments with transient common carotid artery occlusion in mice demonstrated increased levels of OXA and OR1 after ischemia (Nakamachi et al. 2005). In addition, in orexin gene deficient mice permanent focal ischemia led to neuronal cell death, clearly showing the neuroprotective role of OXA and OR1 (Nakamachi et al. 2005).

Cortical cultures in vitro are deprived from orexinergic input, which they normally receive in the brain in vivo. The high expression levels of OXA suggest that cortical cultures may homeostatically switch to the production of OXA as an adaptation mechanism to the isolated environment. A similar phenomenon has been recently described by Corner (2008a), who showed that chronic blockade of either NMDA or AMPA glutamate receptors in organotypic neocortical slices resulted in activation of the other receptor, which maintained spontaneous (bursting) activity of the network. As sufficient activity is vital for their survival (De Marco García et al. 2011), blocking both glutamate receptors would eliminate all activity and cultures would die. However, kainite receptor-mediated bursting

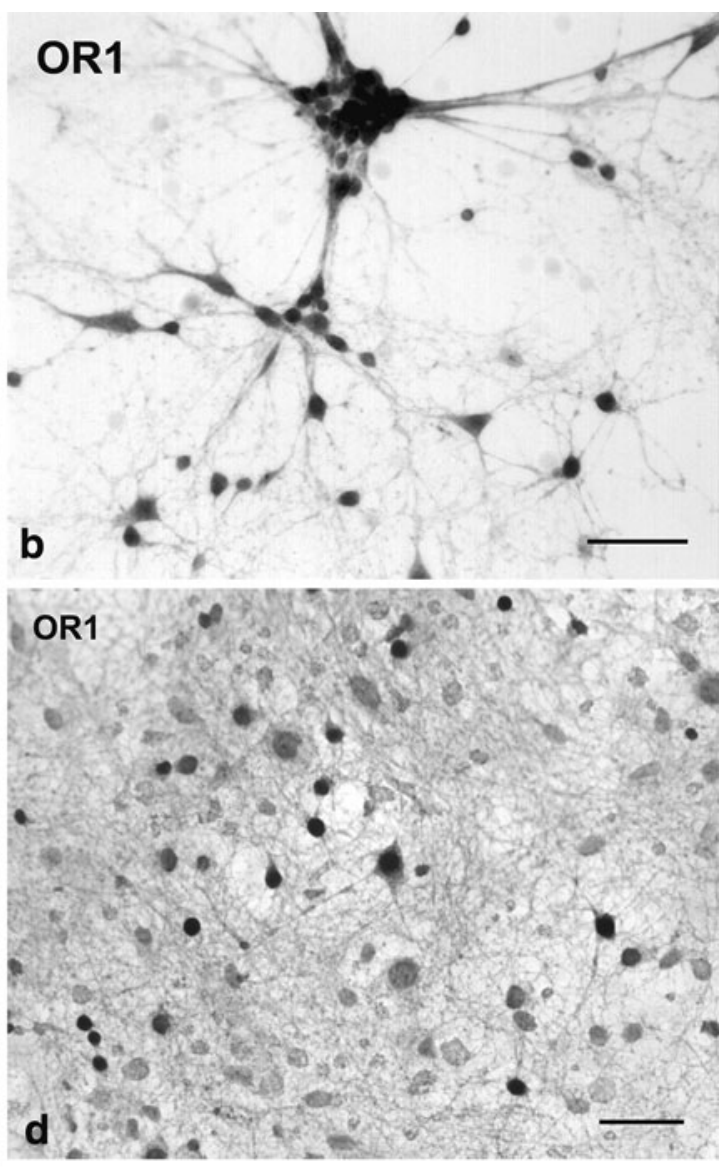

and the reaction product is dispersed also along the neuronal arborizations. Scale bars: (a-c) $30 \mu \mathrm{m}$; (d) $50 \mu \mathrm{m}$

takes over from both of the blocked excitatory receptortypes instead (Corner et al. 2002). When kainite receptors were also blocked, bursts were restored partially because of cholinergic synaptic drive, which cortical neurons normally do not utilize (Corner 2008b). In other words, the developing network is so flexible that it homeostatically switches to another regulatory system or even to another neuronal phenotype in order to survive.

As has been shown, the first 2 weeks after plating are crucial for network formation and survival (Van Pelt et al. 2004). Two major events during this period of time play a significant role in neuronal development: formation of synaptically connected networks, regulated by neurotropic and neurotrophic factors, and the emergence of bioelectrical network-wide activity patterns. In order to survive the axons must have established anatomical and functional connections by day 14 in vitro (Voigt et al. 2005). This neuronal activity follows neural induction (in vivo) and precedes synapse formation (Spitzer 2006). The activity dependence of synapse formation and neuronal survival at later stages of development has been recognized for some 

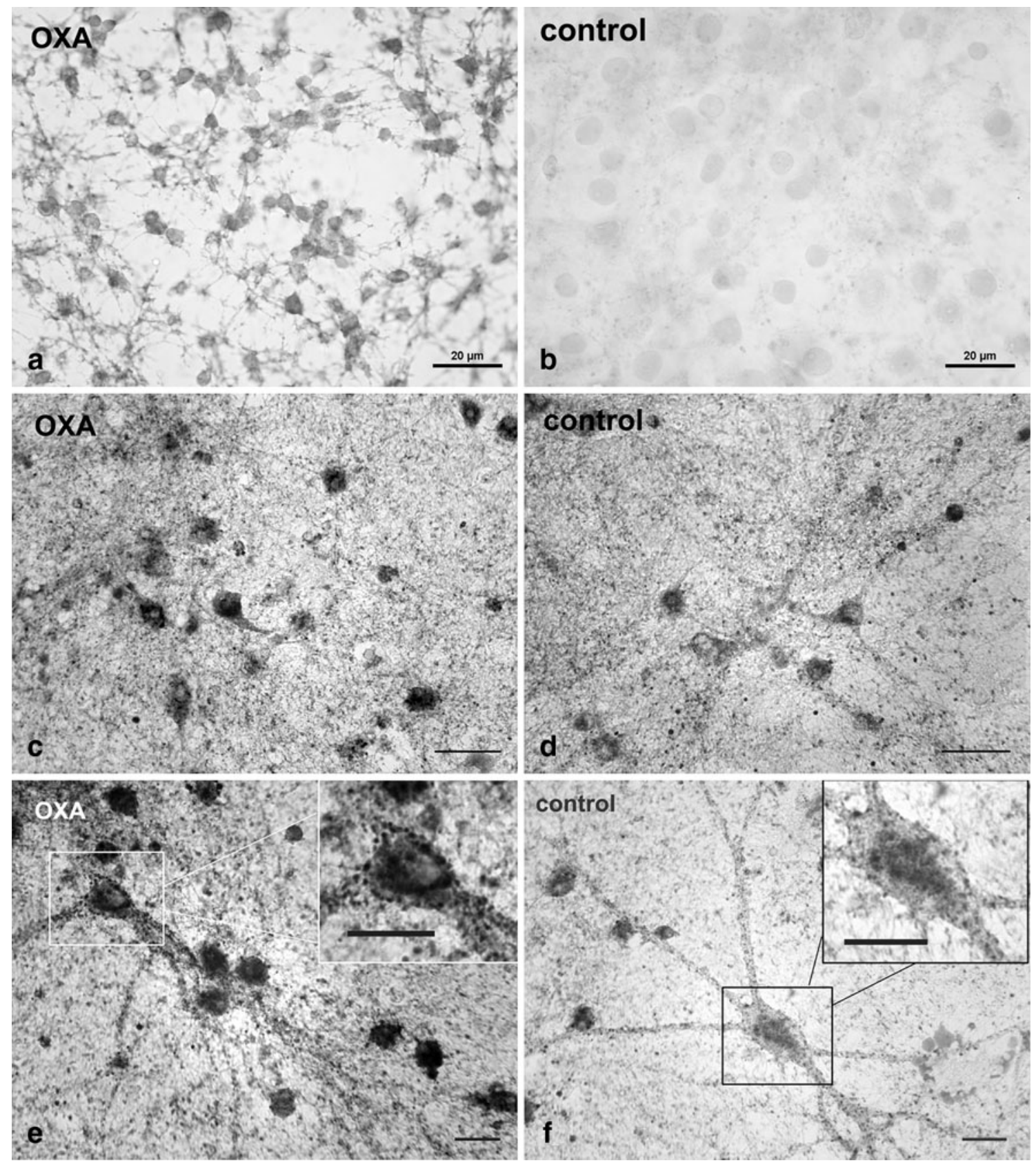

Fig. 4 Immunolabeling for synaptophysin after chronic application of OXA and in the control sister cultures at age of 4 days $(\mathbf{a}, \mathbf{b})$, 2 weeks (c, d), and 3 weeks (e, f). The OXA conditioned cultures show a much higher expression of synaptophysin, earlier onset and

sustained increase beyond 2 weeks in vitro compared to the controls. The panels in e and f show a closer view of fragments of synaptophysin-positive neurons. Notice the higher synaptic density in OXA treated culture. Scale bars: $(\mathbf{a}, \mathbf{b}, \mathbf{e}, \mathbf{f}) 20 \mu \mathrm{m} ;(\mathbf{c}, \mathbf{d}) 30 \mu \mathrm{m}$

time (Zito and Svoboda 2002). Thus, a rapid synapse formation begins during the first week in vitro, and reaches its maximum around day 21 (Ichikawa et al. 1993), as does the activity of the network (Van Pelt et al. 2004; Chiappalone et al. 2006). This qualitatively mimics the in vivo time course of brain development (Romijn et al. 1981), and perfectly coincides with the appearance of the orexinpositive structures in the brain during early postnatal stages (Stoyanova et al. 2010), suggesting that OXA may play a

role in synaptogenesis. Indeed, the results from our experiments with chronic application of OXA to cultured neurons for the first time demonstrate that OXA application has a stimulating effect on synaptogenesis and leads to its early onset.

Thus, in contrast to the in vivo cortex, cortical cultures experience a complete absence of neuronal input. At early developmental stages there is also very little internal activity in cultured networks (see Fig. 2g). However, 
Synaptophysin expression during development

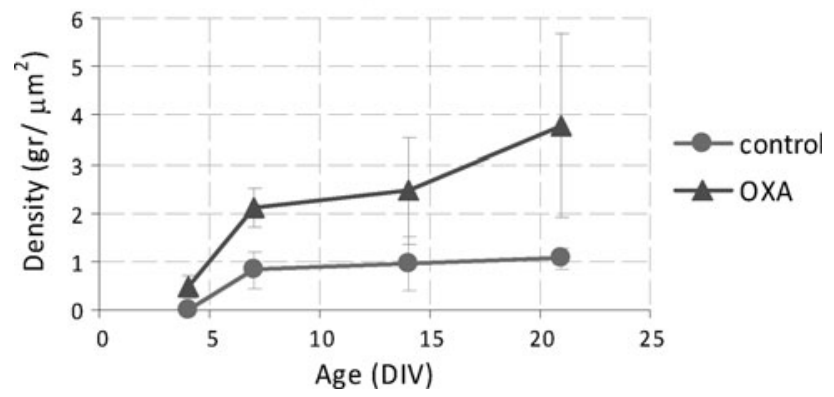

Fig. 5 Development of the number of synaptophysin reactive granules per $\mu \mathrm{m}^{2}$ in control cultures and cultures chronically treated with orexin A during the first 3 weeks of development. All values are shown as mean \pm SEM. The granule density depended significantly on the cultures age, and this density was significantly higher in OXA treated cultures

ongoing activity is crucial for network vitality. The high OXA expression may be a homeostatic reaction to up regulate neuronal activity in isolated cultures. This up regulation may be twofold: OXA has a direct excitatory effect on cortical neurons and synaptogenesis is accelerated. It is yet not clear how these mechanisms interact.

Findings by van Huizen et al. suggested that synaptogenesis may be related to network activity. They achieved accelerated maturation in networks chronically disinhibited by picrotoxin (van Huizen et al. 1987), whereas, classical experiments with tetradotoxin (TTX) blockade of spontaneous neuronal activity in fetal rat neocortex resulted in increased neural outgrowth and retarded synaptogenesis (van Huizen and Romijn 1987).

Electron microscopic investigation of synaptic development in cultured cortical cells of rats showed a decrease in the synaptic density after 14 DIV (Ichikawa et al. 1993). We observed a stabilization of synaptic density around that age in the controls in our study, whereas the density of synaptophysin expression gradually and significantly increased up to 21 DIV in OXA conditioned medium. The fact that the percentage of OXA-positive neurons in our study shows a maturational decrease over the incubation period, reaching its plateau of $32.5 \%$ during the third week in vitro, could be explained by a role as initial stimulator of synaptogenesis required for establishment of functional connections and survival of the network.

Our hypothesis that OXA is important in network formation is further supported by the results of studies by Van den Pol et al. and Li et al., who showed that orexinergic neurons in the hypothalamus are mainly innervated by excitatory glutamatergic synapses (Van den Pol et al. 1990, 2001), and have a unique synaptic architecture: they are driven by glutamatergic inputs from local neurons that are utilized by orexinergic neurons to form positive feedback circuits onto themselves ( $\mathrm{Li}$ et al. 2002). Thus, at the early stages of network development, when excitatory input is crucial (Mennerick and Zorumski 2000; De Marco García et al. 2011), the neurons are able to establish regulatory loops for self-stimulation (and thus survival) via onset of OXA synthesis. Because of the strong mutual influences between neuronal connectivity and activity, and the development of connectivity, already shown at network level (le Feber et al. 2009, 2010), further electrophysiological experiments may functionally validate the effects of OXA on the network activity.

In summary, dissociated postnatal cortical neurons of the rat express OXA, which could be regarded as a form of homeostatic neuronal plasticity, an adaptation mechanism to a new environment. Our findings demonstrate for the first time that, in vitro, OXA stimulates synaptogenesis and thus neuronal interactions. This implies involvement of the neurotransmitter in network development and maturation during the early stages of culturing. Whether this effect is direct or exerted via different neurotrophic factors still remains to be elucidated.

Acknowledgments We thank Karin Groot Jebbink and Bettie Klomphaar for their assistance in cell culturing. This work was supported by the EU project NEURoVERS-it (grant MRTN-CT-2005019247).

\section{Conflict of interest None.}

Open Access This article is distributed under the terms of the Creative Commons Attribution Noncommercial License which permits any noncommercial use, distribution, and reproduction in any medium, provided the original author(s) and source are credited.

\section{References}

Ben-Ari Y, Tseeb V, Raggozzino D, Khazipov R, Gaiarsa JL (1994) Gamma-aminobutyric acid (GABA) a fast excitatory transmitter, which may regulate the development of hyppocampal neurons in early postnatal life. Prog Brain Res 102:261-273

Chiappalone M, Bove M, Vato A, Tedesco M, Martinoia S (2006) Dissociated cortical networks show spontaneously correlated activity patterns during in vitro development. Brain Res 1093:41-53

Corner MA (2008a) Reciprocal homeostatic responses to excitatory synaptic receptor inactivation in developing organotypic cortical networks in vitro. Neurosci Lett 438:300-302

Corner MA (2008b) Spontaneous neuronal burst discharges as dependent and independent variables in the maturation of cerebral cortex tissue cultured in vitro: a review of activitydependent studies in live 'model' systems for the development of intrinsically generated bioelectric slow-wave sleep patterns. Brain Res Rev 59:221-244

Corner MA, van Pelt J, Wolters PS, Baker RE, Nuytinck RH (2002) Physiological effects of sustained blockade of excitatory synaptic transmission on spontaneously active developing neuronal networks-an inquiry into the reciprocal linkage between intrinsic biorhythms and neuroplasticity in early ontogeny. Neurosci Biobehav Rev 26:127-185 
Cui LB, Li BW, Jin XH, Zhao L, Shi J (2010) Progressive changes of orexin system in a rat model of 6-hydroxydopamine-induced Parkinson's disease. Neurosci Bull 26:381-387

De Lecea L, Kilduff TS, Peyron C, Gao X-B, Foye PE, Danielson PE, Fukuhara C, Battenberg ELF, Gautvik VT, Bartkett FS, Frankel WN, van den Pol AN, Bloom FE, Gauthvik KM, Sutcliffe JG (1998) The hypocretins: hypothalamus-specific peptides with neuroexitatory activity. Proc Natl Acad Sci USA 95:322-327

De Marco García NV, Karayannis T, Fishell G (2011) Neuronal activity is required for the development of specific cortical interneuron subtypes. Nature. doi:10.1038/nature09865

Fadel J, Bubser M, Deutch AY (2002) Differential activation of orexin neurons by antipsychotic drugs associated with weight gain. J Neurosci 22:6742-6746

Friedman LF, Zeitzer JM, Lin L, Hoff D, Mignot E, Peskind ER, Yesavage JA (2007) In Alzheimer disease, increased wake fragmentation found in those with lower hypocretin-1. Neurology 68:793-794

Fronczek R, Overeem S, Lee SYY, Hegeman IM, van Pelt J, van Duinen SG, Lammers GJ, Swaab DF (2007) Hypocretin (orexin) loss in Parkinson's disease. Brain 130:1577-1585

Gautvik KM, de Lecea L, Gautvik VT, Danielson PE, Tranque P, Dopazo A, Bloom FE, Sutcliffe JG (1996) Overview of the most prevalent hypothalamus-specific mRNAs, as identified by directional tag PCR substraction. Proc Natl Acad Sci USA 93:8733-8738

Horvath TL, Gao XB (2005) Input organization and plasticity of hypocretin neurons: possible clues to obesity's association with insomnia. Cell Metab 1:279-286

Horvath TL, Peyron C, Diano S, Ivanov A, Aston-Jones G, Kilduff TS, van den Pol AN (1999) Hypocretin (Orexin) activation and synaptic innervation of the locus coeruleus noradrenergic system. J Comp Neurol 415:145-159

Hsu SM, Raine L, Fanger H (1981) Use of avidin-biotin-peroxidase complex $(\mathrm{ABC})$ in immunoperoxidase techniques: a comparison between $\mathrm{ABC}$ and unlabeled antibody (PAP) procedures. J Histochem Cytochem 29:577-580

Ichikawa M, Muramoto K, Kobayashi K, Kawahara M, Kuroda Y (1993) Formation and maturation of synapses in primary cultures of rat cerebral cortical cells: an electron microscopic study. Neurosci Res 16:95-103

le Feber J, Van Pelt J, Rutten W (2009) Latency related development of functional connections in cultured cortical networks. Biophys J 96:3443-3450

le Feber J, Stegenga J, Rutten WLC (2010) The effect of slow electrical stimuli to achieve learning in cultured networks of rat cortical neurons. PLoS ONE 5:e8871

Levitt P, Harvey JA, Simansky K, Murphy EH (1997) New evidence for neurotransmitter influences on brain development. Trends Neurosci 20:269-274

Li Y, Gao XB, Sakurai T, van Pol AN (2002) Hypocretin/orexin excites hypocretin neurons via a local glutamate neuron-a potential mechanism for orchestrating the hypothalamic arousal system. Neuron 36:1169-1181

Ljungdahl A, Hokfelt T, Nilsson G (1978) Distribution of substance P-like immunoreactivity in the central nervous system of the ratI. Cell bodies and nerve terminals. Neuroscience 3:861-943

Mennerick S, Zorumski CF (2000) Neural activity and survival in the developing nervous system. Mol Neurobiol 22:41-54

Nakamachi T, Endo S, Ohtaki H, Yin L, Kenji D, Kudo Y, Funahashi H, Matsuda K, Shioda S (2005) Orexin-1 receptor expression after global ischemia in mice. Regul Pept 126:49-54

Peever JH, Lai YY, Siegel JM (2003) Excitatory effects of hypocretin-1 (orexin-A) in the trigeminal motor nucleus are reversed by NMDA antagonism. J Neurophysiol 89:2591-2600
Petersén A, Gil J, Maat-Schieman ML, Björkqvist M, Tanila H, Araújo IM, Smith R, Popovic N, Wierup N, Norlén P, Li JY, Roos RA, Sundler F, Mulder H, Brundin P (2005) Orexin loss in Huntington's disease. Hum Mol Genet 14:39-47

Ripley B, Overeem S, Fujiki N, Nevsimalova S, Uchino M, Yesavage J, Di Monte D, Dohi K, Melberg A, Lammers GJ, Nishida Y, Roelandse FW, Hungs M, Mignot E, Nishino S (2001) CSF hypocretin/orexin levels in narcolepsy and other neurological conditions. Neurology 57:2253-2258

Romijn HJ, Habets MT, Mud MT, Wolters PS (1981) Nerve outgrowth, synaptogenesis and bioelectric activity in fetal rat cerebral cortex tissue cultured in serum-free, chemically defined medium. Develop Brain Res 2:583-589

Romijn HJ, van Huizen F, Wolters PS (1984) Towards an improved serum-free, chemically defined medium for long-term culturing of cerebral cortex tissue. Neurosci Biobehav Rev 8:301-334

Sakurai T, Ameliya A, Isgii M, Matsuzaki I, Chemelli RM, Tanaka H, Williams SC, Richardson JA, Kozlowski GP, Wilson S, Arch JRS, Buckingham RE, Haysen AC, Carr SA, Annan RS, McNulty DE, Liu W-S, Terrett JA, Elshourbagy NA, Bergsma DJ, Yanagisawa M (1998) Orexins and orexin receptors: a family of hypothalamic neuropeptides and $G$ protein-coupled receptors that regulate feeding behavior. Cell 92:573-585

Spitzer NC (2006) Electrical activity in early neuronal development. Nature 444:707-712

Spoerri P (1988) Neurotrophic effect of GABA in cultures of embryonic chick brain and retina. Synapse 2:11-22

Stoyanova II, Rutten WLC, le Feber J (2010) Orexin-A and orexin-B during the postnatal development of the rat brain. Cell Mol Neurobiol 30:81-89

Stoyanova II, le Feber J, Rutten WLC (2011) Acute stimulation of dissociated cortical neurons of newborn rats with Orexin A: effect on the network activity. In: El Haj A, Bader D (eds) IFMBE proceedings, 8th international conference on cell \& stem cell engineering (ICCE), June 11-12, 2010 Ireland, vol. 30, pp 35-38, ISBN: 978-3-642-19043-8

Trivedi P, Yu H, MacNeil DJ, Van der Ploeg LTH, Guan XM (1998) Distribution of orexin receptor mRNA in the rat brain. FEBS Lett 438:71-75

Van den Pol AN, Patrylo PR, Ghosh PK, Gao XB (2001) Lateral hypothalamus: early developmental expression and response to hypocretin (orexin). J Comp Neurol 433:349-363

Van den Pol AN, Wuarin JP, Dudek FE (1990) Glutamate, the dominant excitatory transmitter in neuroendocrine regulation. Science 250:1276-1278

van Huizen F, Romijn HJ (1987) Tetradotoxin enhances initial neurite outgrowth from fetal rat cerebral cortex cells in vitro. Brain Res 408:271-274

van Huizen F, Romijn HJ, Habets AM, van den Hoof P (1987) Accelerated neural network formation in rat cerebral cortex cultures chronically disinhibited with Picrotoxin. Exp Neurology 97:280-288

Van Pelt J, Corner MA, Wolters PS, Rutten WLC, Ramakers GJA (2004) Longterm stability and developmental changes in spontaneous network burst firing patterns in dissociated rat cerebral cortex cell cultures on multielectrode arrays. Neurosci Lett 361:86-89

Voigt T, Opitz T, de Lima AD (2005) Activation of early silent synapses by spontaneous synchronous network activity limits the range of network connections. J Neurosci 25:4605-4615

Zito K, Svoboda K (2002) Activity-dependent synaptogenesis in the adult mammalian cortex. Neuron 35:1015-1017 